\title{
Memoria auditiva y rendimiento neuropsicológico en pacientes de la tercera edad
}

\author{
Auditory memory and neuropsychological performance in elderly patients
}

IGOR INFANTES $^{1}$

Centro Geriátrico y Gerontológico de la Fuerza Armada del Perú (FAP)

(RECIBIDO 05//02/2015, AcEPTADO 20/06/2015)

\begin{abstract}
RESUMEN
Objetivo: Determinar la relación entre la memoria auditiva y el rendimiento neuropsicológico en pacientes de la tercera edad atendidos en el Centro Geriátrico y Gerontológico de la FAP. Diseño: La investigación es de tipo aplicada, mientras que el diseño es de tipo observacional retrospectivo y de corte transversal, con un nivel de estudio descriptivo correlacional. Institución: Centro Geriátrico y Gerontológico de la Fuerza Aérea del Perú. Material: Historias clínicas de pacientes atendidos en la consulta externa del Servicio de Neuropsicología. Métodos: Se evaluaron 212 historias clínicas de entre los años 2012 y 2014, mediante muestreo aleatorio estratificado. Principales medidas de resultados: Memoria auditiva y rendimiento neuropsicológico en el paciente de la tercera edad. Resultados: Los pacientes con demencia tipo Alzheimer presentaron una puntuación media en memoria auditiva de 5.17; con demencia vascular, 6.75; con demencia mixta, 4.93; y los pacientes sanos, 10.05. Conclusiones: Existe relación positiva entre la memoria auditiva y el rendimiento neuropsicológico en pacientes de la tercera edad atendidos en el Centro Geriátrico y Gerontológico de la FAP. Asimismo, se encontró que existen diferencias significativas en las puntuaciones totales en memoria auditiva, de acuerdo al diagnóstico de demencia, en los pacientes de la muestra investigada.
\end{abstract}

Palabras clave: Memoria auditiva, rendimiento neuropsicológico, pacientes de la tercera edad.

\begin{abstract}
Objective: To determine the relationship between auditory memory and neuropsychological performance in elderly patients treated at the Geriatric and Gerontology Center of FAP. Design: The research is applied type, while the design is retrospective observational and cross-sectional, with a descriptive correlational level. Institution: Geriatric and Gerontology Center of the Air Force of Peru. Material: Medical records of patients seen in the outpatient Department of Neuropsychology. Methods: 212 clinics between 2012 and 2014 stories were assessed by stratified random sampling. Main outcome measures: Auditory memory
\end{abstract}

1 Psicólogo del Centro Geriátrico y Gerontológico de la Fuerza Aérea del Perú. E-mail: igorinfantes@hotmail.com 
and neuropsychological performance in patients older. Results: Patients with Alzheimer's dementia showed a mean score of 5.17 auditory memory; with vascular dementia, 6.75; with mixed dementia, 4.93; and healthy, 10.05 patients. Conclusions: There is a positive relationship between auditory memory and neuropsychological performance in elderly patients treated at the Geriatric and Gerontology Center of FAP. We also found significant differences in total scores on auditory memory, according to the diagnosis of dementia in patients of the investigated sample.

Keywords: Auditory memory, neuropsychological performance, elderly patients.

\section{INTRODUCCIÓN}

Los problemas de memoria suelen ser la queja cognitiva más frecuente de los pacientes con síndromes neuropsicológicos (síndromes conductuales de naturaleza orgánica). La pérdida de información, el olvido de tareas relacionadas con el trabajo o la dificultad para recordar compromisos adquiridos con otras personas pueden proporcionar importantes perjuicios en el ámbito social y laboral, especialmente si no se ha diagnosticado la naturaleza del problema de memoria. Una adecuada identificación de los problemas de memoria que puede presentar una persona posibilita que, tanto desde el punto de vista clínico como familiar, se puedan emprender las estrategias adecuadas para ayudar a la persona y evitar graves consecuencias personales. Diferentes afecciones neurológicas pueden producir diferentes tipos de alteraciones en la memoria, cada una de las cuales se encuentra relacionada con diferentes mecanismos fisiopatológicos. No obstante, hay que tener en cuenta que no todas las dificultades en la memoria tienen una causa neurológica. Diferentes condiciones psiquiátricas, así como estados depresivos, ansiosos o emocionales, pueden producir alteraciones en la memoria. Un diagnóstico equivocado, por ejemplo, de depresión en vez de demencia, o a la inversa, puede llevar a meses, e incluso años, de tratamiento inadecuado.

Desde un punto de vista práctico-clínico, se considera la memoria como la capacidad del individuo para almacenar información y recuperarla más tarde. Esta capacidad tiene un importante valor adaptativo, por lo que cuando se altera o se pierde la capacidad de adaptación del individuo se ve considerablemente afectada.

Es necesario tener presente aspectos como la discriminación de la naturaleza de las dificultades de memoria, es decir, discriminar si la alteración de la memoria es un problema específico relacionado con los sistemas de memoria o es un problema relacionado con otros sistemas cognitivos. Así, por ejemplo, muchas personas pueden manifestar que tienen problemas de memoria cuando lo único que presentan son dificultades para evocar palabras comunes o nombres propios (disnomia), mientras que su capacidad para recuperar memorias episódicas se encuentra perfectamente. Es el caso de la memoria auditiva, que es la capacidad para evocar y recordar sonidos escuchados. Se puede clasificar en memoria auditiva a corto plazo y en memoria auditiva a largo plazo. En el caso de la de corto plazo, esta hace referencia a la capacidad de recordar la información auditiva durante un breve periodo de tiempo (varios segundos). En comparación con la 
memoria visual y sensorial, se cree que la memoria auditiva dura un poco más, alrededor de tres o cuatro segundos. Y en cuanto a la memoria auditiva a largo plazo, esta puede retener el recuerdo durante unos días.

El rendimiento de la memoria de las personas mayores ha sido comparado con el que padecen las personas con lesiones en el diencéfalo (Reuter-Lorenz, 2002), aunque los efectos físicos del envejecimiento normal no son tan dramáticos y las consecuencias funcionales son menores. La capacidad para detectar pacientes con alta probabilidad de desarrollar la enfermedad de Alzheimer, de entre los que presentan una mínima alteración cognitiva, aumenta cuando los datos de edad y función mnésica se combinan con medidas de atrofia del lóbulo temporal (Visser, Verhey, Hofman, Scheltens y Jolles, 2002). La literatura aporta una imagen muy consistente acerca de la influencia del envejecimiento en la memoria: las diferencias por edad son mínimas en tareas de memoria procedimental o implícita, muy leves en tareas de memoria semántica e importantes en tareas de memoria episódica (Craik \& Jennings, 1992). Un estudio reportó que los grupos con enfermedad de Alzheimer y demencia semántica presentaban un deterioro significativo, con relación al grupo con demencia frontotemporal, en memoria verbal, mientras que solo el grupo con enfermedad de Alzheimer tenía deterioro en memoria visual (Kramer, Jurik, Sha et al., 2003). Los pacientes con demencia frontotemporal rindieron significativamente peor en extensión de dígitos hacia atrás y cometieron significativamente más errores ejecutivos que los pacientes de la enfermedad de Alzheimer y de demencia semántica. Los pacientes con demencia semántica presentaban más deterioro que aquellos con enfermedad de Alzheimer y demencia frontotemporal en nombres de confrontación.

En pacientes diagnosticados con algún tipo de demencia se suele evaluar el funcionamiento de aquellas capacidades (variables de la actividad mental) que pueden afectar el rendimiento mnésico, tales como atención, concentración, velocidad de procesamiento de la información, organización, estrategias, esfuerzo y motivación.

Por tal razón, el propósito de este estudio ha sido determinar las características de la memoria auditiva y su grado de asociación con el rendimiento neuropsicológico en pacientes de la tercera edad con envejecimiento normal y demencias tipo Alzheimer, vascular y mixta, que son atendidos en un Centro Geriátrico y Gerontológico de Lima Metropolitana. Los resultados permitirán contar con una curva de aprendizaje acorde al grado de rendimiento neuropsicológico observado en el contexto social y geográfico de los pacientes.

\section{MÉTODO}

La investigación es de tipo aplicada, con diseño observacional retrospectivo y de corte transversal; mientras que el nivel del estudio es descriptivo correlacional (Hernández, Fernández y Baptista, 2010). Asimismo, los datos fueron recogidos de las historias clínicas, donde se registran los resultados de las evaluaciones neu- 
ropsicológicas que se realizaron entre los años 2012 y 2014 a pacientes adultos mayores que son atendidos en el Centro Geriátrico y Gerontológico de la FAP, y que presentan diagnóstico de deterioro neuropsicológico normal o con alguna de las demencias: tipo Alzheimer, vascular o mixta; por lo que la selección de los participantes fue de manera censal. De este modo, se trabajó con 212 pacientes distribuidos en cuatro grupos, de acuerdo al diagnóstico encontrado.

Los criterios de selección de los participantes serán los siguientes:

\section{Criterios de inclusión}

- Pacientes mayores de 60 años de edad.

- Pacientes diagnosticados con deterioro neuropsicológico normal o con alguna de las siguientes demencias: tipo Alzheimer, vascular o mixta.

\section{Criterios de exclusión}

- Pacientes menores de 60 años de edad.

- Pacientes diagnosticados con otras demencias, tales como demencia frontotemporal y demencia con cuerpos de Lewy en comorbilidad con demencias tipo Alzheimer, vascular y mixta.

- Pacientes con politraumatismos encéfalo craneanos.

Los instrumentos utilizados son: El Test de Aprendizaje Auditivo-Verbal de Rey (Rey, 1964), y el NEUROPSI (Evaluación Psicológica Breve en Español) (Ostrosky, Ardila y Rosselli, 1999).

Se coordinó y obtuvo la autorización de la Dirección del Centro Geriátrico y Gerontológico de la FAP, a efectos de contar con el permiso para acceder a las historias y realizar el registro de los datos de la batería de pruebas neuropsicológicas administradas a los pacientes de la tercera edad que fueron atendidos en consulta entre los años 2012 y 2014, manteniendo la confidencialidad de los datos consignados.

\section{RESULTADOS}

Tabla 1. Correlación entre las variables aprendizaje auditivo-verbal y rendimiento neuropsicológico

\begin{tabular}{lcc}
\hline & & Rendimiento neuropsicológico \\
\hline & Correlación de Pearson & $0.761^{* *}$ \\
Aprendizaje auditivo-verbal (total) & Sig. (unilateral) & 0.000 \\
& $\mathrm{~N}$ & 212 \\
\hline
\end{tabular}

** Significativa al nivel de $\mathrm{p}<0.01$

El valor obtenido de la correlación entre las variables de estudio es un valor estadísticamente significativo al nivel de $\mathrm{p}<0.01(\mathrm{r}=0.761$; Sig. unilateral $=$ 
0.000). De acuerdo a tales resultados, se puede establecer que las puntuaciones en la variable Memoria inmediata correlacionan positivamente con las puntuaciones de la variable Rendimiento neuropsicológico.

Tabla 2. Correlación entre la dimensión Memoria inmediata y atención verbal con el rendimiento neuropsicológico

\begin{tabular}{ccc}
\hline & & Rendimiento neuropsicológico \\
\hline \multirow{2}{*}{ Memoria inmediata y atención } & Correlación de Pearson & $0.725^{* *}$ \\
verbal & Sig. (unilateral) & 0.000 \\
& $\mathrm{~N}$ & 212 \\
\hline
\end{tabular}

** Significativa al nivel de $\mathrm{p}<0.01$.

El valor obtenido de la correlación entre la dimensión Memoria inmediata y atención verbal con el rendimiento neuropsicológico es un valor estadísticamente significativo al nivel de $\mathrm{p}<0.01(\mathrm{r}=0.725$; Sig. unilateral $=0.000)$. En tal sentido, se puede afirmar que las puntuaciones en la dimensión Memoria inmediata $y$ atención verbal correlacionan positivamente con las puntuaciones de la variable Rendimiento neuropsicológico.

Tabla 3. Correlación entre la dimensión Retención a corto y largo plazo y memoria verbal con el Rendimiento neuropsicológico

\begin{tabular}{ccc}
\hline & & Rendimiento neuropsicológico \\
\hline \multirow{2}{*}{ Retención a corto y largo plazo y } & Correlación de Pearson & $0.741^{* *}$ \\
memoria verbal & Sig. (unilateral) & 0.000 \\
& $\mathrm{~N}$ & 212 \\
\hline
\end{tabular}

** Significativa al nivel de $\mathrm{p}<0,01$.

La correlación entre la dimensión Retención a corto y largo plazo y memoria verbal con el rendimiento neuropsicológico se traduce en un valor estadísticamente significativo al nivel de $\mathrm{p}<0.01(\mathrm{r}=0.741 ;$ Sig. unilateral $=0.000)$. De manera que se puede sostener que las puntuaciones en la dimensión Retención a corto y largo plazo y memoria verbal correlacionan positivamente con las puntuaciones de la variable Rendimiento neuropsicológico.

Tabla 4. Correlación entre la dimensión Aprendizaje y evocación con el rendimiento neuropsicológico

\begin{tabular}{ccc}
\hline & & Rendimiento neuropsicológico \\
\hline & Correlación de Pearson & $0.758^{* *}$ \\
Aprendizaje y evocación & Sig. (unilateral) & 0.000 \\
& $\mathrm{~N}$ & 212 \\
\hline
\end{tabular}

** Significativa al nivel de $\mathrm{p}<0.01$. 
La correlación entre la dimensión Aprendizaje y evocación con el rendimiento neuropsicológico arroja un valor estadísticamente significativo al nivel de $\mathrm{p}<0.01$ ( $\mathrm{r}=0.758$; Sig. unilateral $=0.000)$. De manera que se puede afirmar que las puntuaciones en la dimensión Aprendizaje y evocación correlacionan positivamente con las puntuaciones de la variable Rendimiento neuropsicológico.

Con el uso de la prueba estadística ANOVA, se obtuvo un valor $\mathrm{F}=38.015$, con una Sig. $=0.000$, lo cual indica que las diferencias en la dimensión Memoria inmediata y atención verbal, de la variable Memoria auditiva, son significativas al nivel de $\mathrm{p}<0.01$, de acuerdo al diagnóstico de demencia. Se encontró, además, que las más altas puntuaciones medias corresponden al grupo de pacientes sanos, mientras que las más bajas, al grupo de pacientes con demencia mixta (Figura 1).

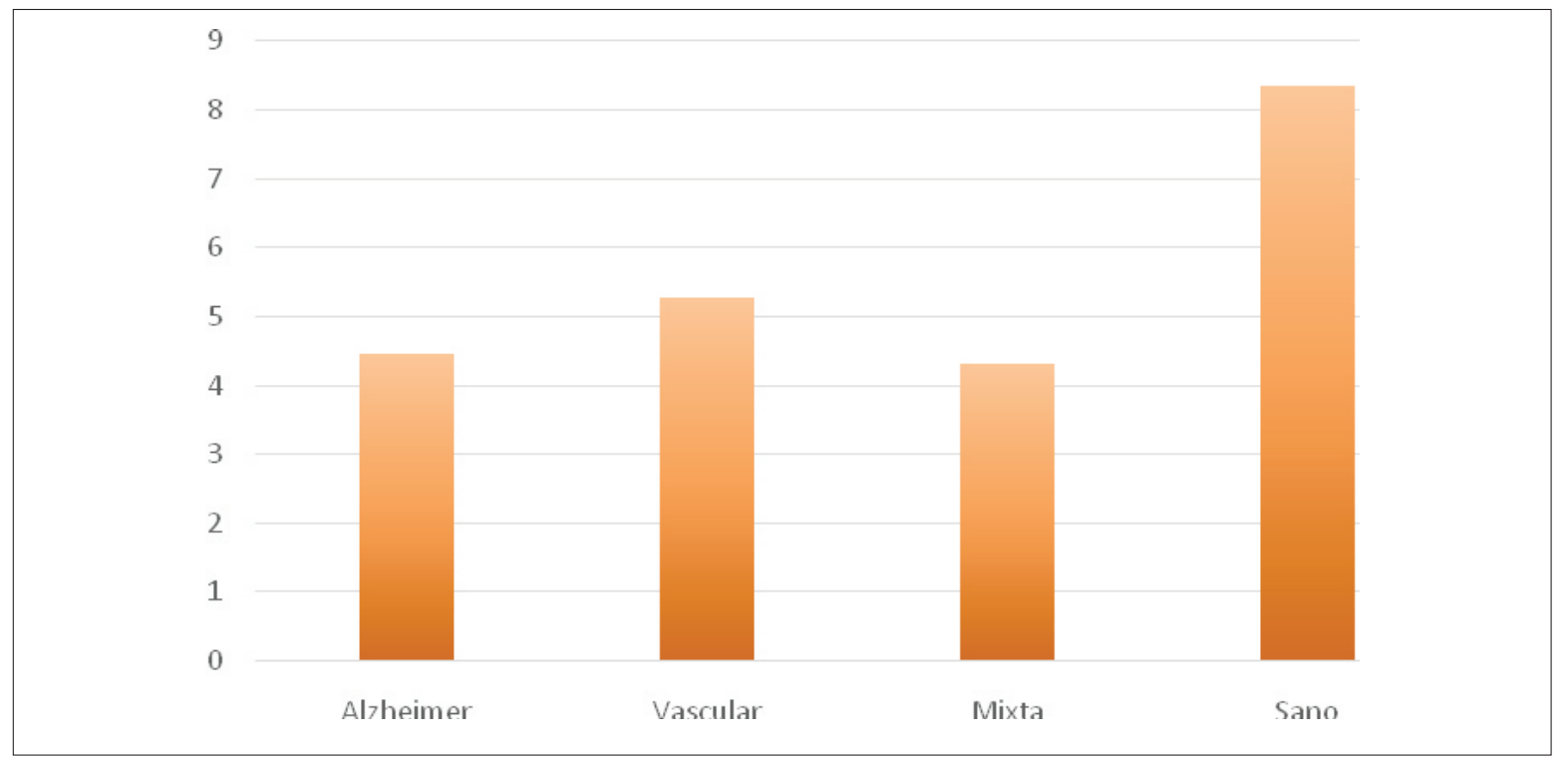

Figura 1. Diferencias en la dimensión Memoria inmediata y atención verbal, de acuerdo al diagnóstico de demencia

Mediante la prueba estadística ANOVA, se obtuvo un valor $\mathrm{F}=41.694$, con una Sig. $=0.000$, lo cual indica que las diferencias en la dimensión Retención a corto y largo plazo y memoria verbal, de la variable Memoria auditiva, son significativas al nivel de $\mathrm{p}<0.01$, considerando el diagnóstico de demencia. Se encontró, asimismo, que las más altas puntuaciones medias corresponden al grupo de pacientes sanos, mientras que las más bajas, al grupo de pacientes con demencia mixta (Figura 2). 


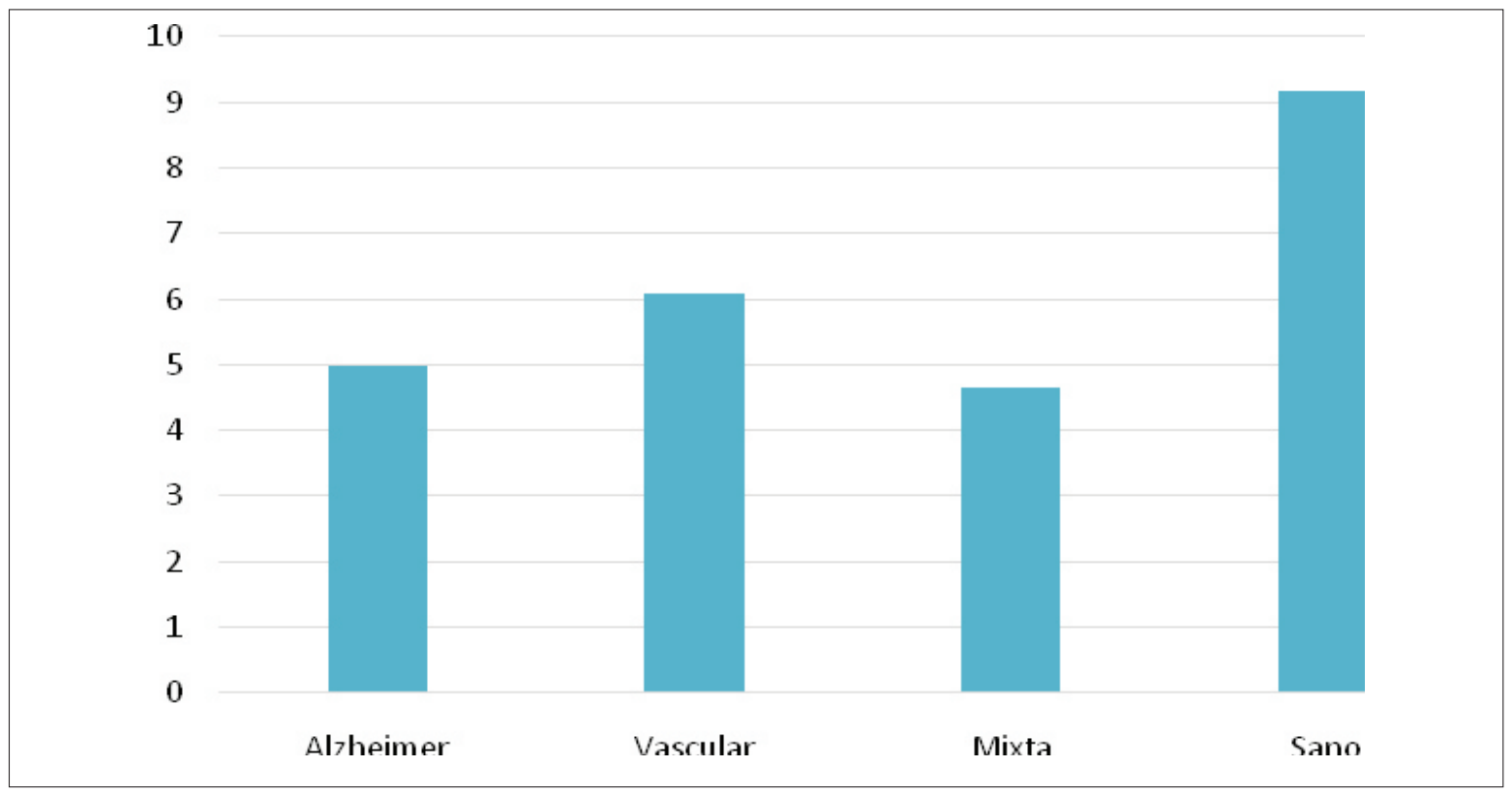

Figura 2. Diferencias en la dimensión Retención a corto y largo plazo y memoria verbal, de acuerdo al diagnóstico de demencia.

Mediante la aplicación de la prueba estadística ANOVA, se obtuvo un valor $\mathrm{F}=$ 52.400 , con una Sig. $=0.000$, lo cual indica que las diferencias en la dimensión Aprendizaje y evocación, de la variable Memoria auditiva, son significativas, considerando el diagnóstico de demencia. Se encontró, además, que las más altas puntuaciones medias corresponden al grupo de pacientes sanos, mientras que las más bajas, al grupo de pacientes con demencia mixta (Figura 3).

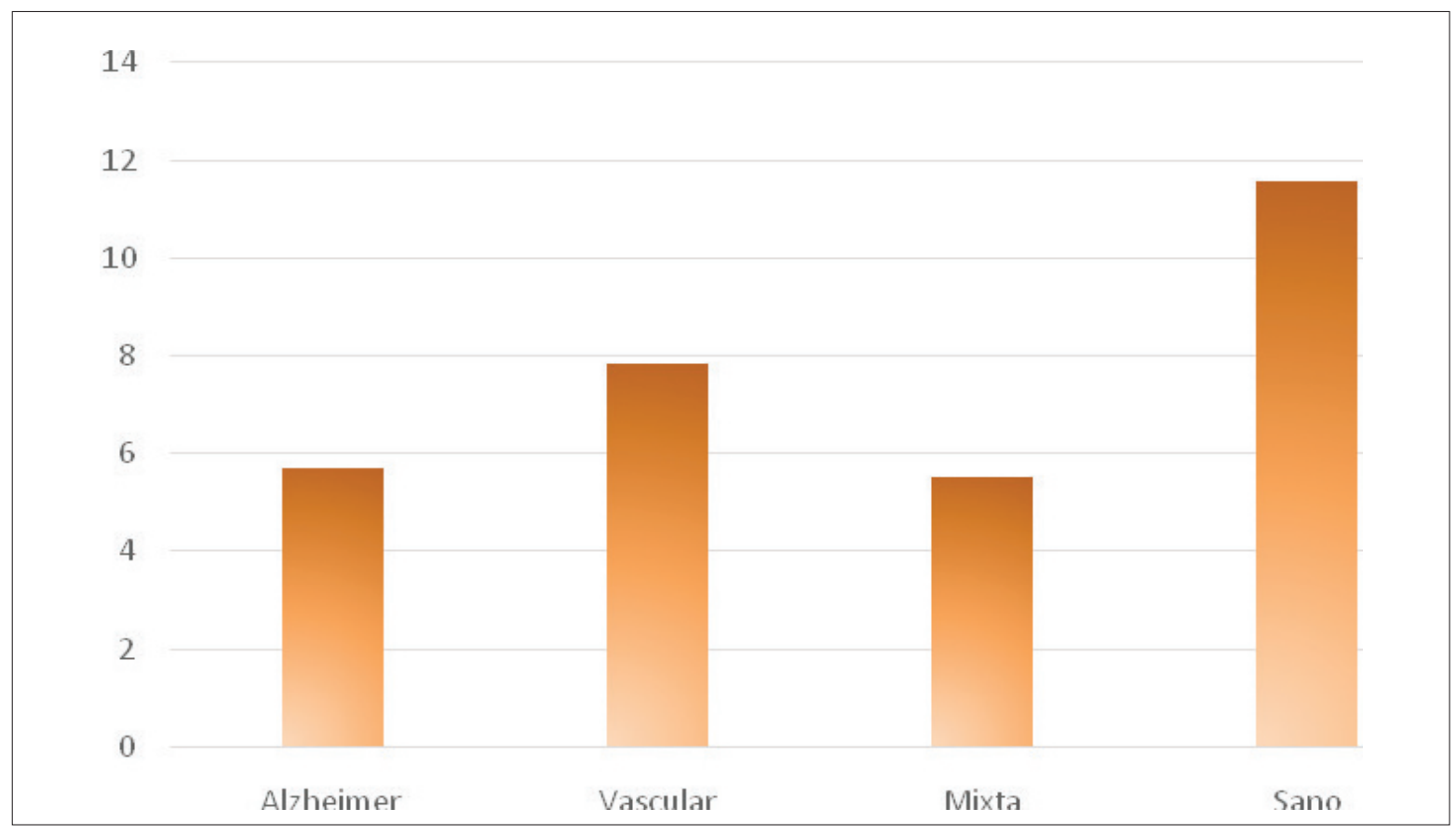

Figura 3. Diferencias en la dimensión Aprendizaje y evocación, de acuerdo al diagnóstico de demencia 
$\mathrm{Al}$ aplicar la prueba estadística ANOVA, se obtuvo un valor $\mathrm{F}=49.541$, con una Sig. $=0.000$, lo cual indica que las diferencias en la variable Memoria auditiva son significativas, considerando el diagnóstico de demencia. Se encontró, además, que las más altas puntuaciones medias corresponden al grupo de pacientes sanos, mientras que las más bajas, al grupo de pacientes con demencia mixta (Figura 4).

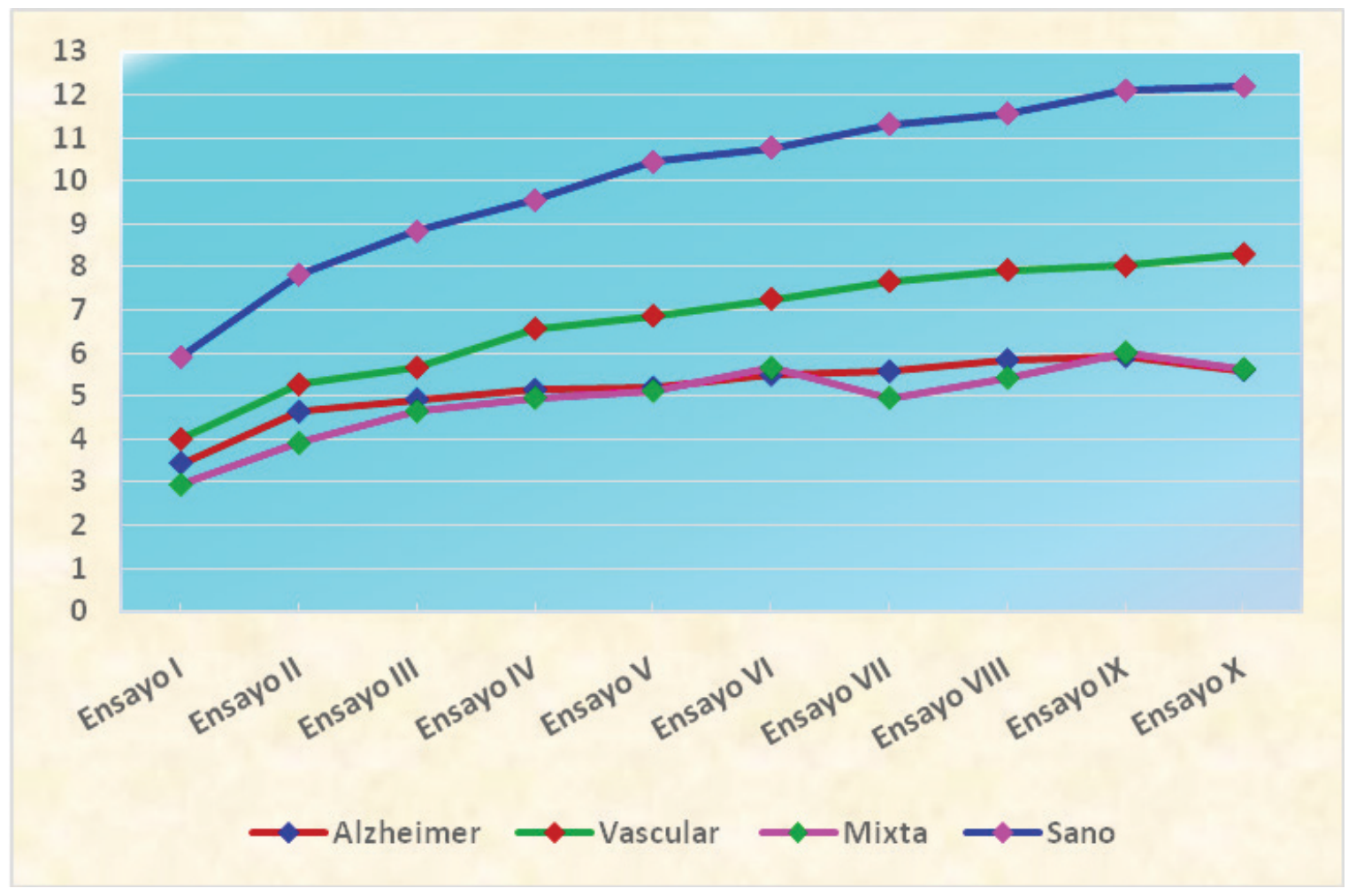

Figura 4. Dispersigrama de las puntuaciones medias en Memoria auditiva 


\section{DISCUSIÓN}

En los últimos años, con el incremento de la esperanza de vida en la población de adultos mayores, la calidad de vida en la tercera edad se ha vuelto una preocupación de salud pública, sobre todo, por la aparición cada vez frecuente de enfermedades neuropsicológicas que dan a lugar cuadros preclínicos y clínicos de diversas demencias y pseudodemencias, como consecuencia del deterioro de la memoria, entre otros factores.

Es bien conocida en nuestro medio la demencia tipo Alzheimer (DTA), su evolución y consecuencias; también, lo mismo que la DTA, se presentan muchos casos de demencia vascular y demencia mixta. Los resultados obtenidos en esta investigación permiten afirmar que entre la memoria auditiva y el rendimiento neuropsicológico existe una fuerte relación, encontrando además que los pacientes adultos mayores con DTA presentan un mayor deterioro tanto en su memoria auditiva como en las principales áreas neuropsicológicas (codificación, funciones ejecutivas y funciones de evocación) evaluadas con el Neuropsi. En efecto, los pacientes con DTA no pueden codificar adecuadamente la información, a pesar de tratar de facilitárselo con descriptores semánticos, y obtienen menor beneficio de las claves semánticas durante la evocación, por lo que no parece que la causa principal del pobre rendimiento en aprendizaje sea un problema de acceso a la información, sino de su codificación. Los pacientes de este grupo presentan, pues, una curva de aprendizaje que refleja una pobrísima ejecución de sus funciones mnésicas, tanto de la memoria inmediata como de la evocación de un listado de palabras, que se incluye en el Test Auditivo-Verbal de Rey (Gramunt, 2008).

El grupo de pacientes sanos, que ha hecho las veces de grupo de control, es el que sale mejor librado en las evaluaciones tanto de la memoria auditiva como de sus funciones neuropsicológicas. Es decir, los pocos déficit hallados, tanto mnésicas como neuropsicológicas, pueden atribuirse a un deterioro normal como consecuencia del envejecimiento. Cabe mencionar, asimismo, que el grupo de pacientes con demencia vascular presentan ya una disminución de algunas de sus funciones mnésicas y neuropsicológicas; pero, no obstante, no son tan acentuadas como sucede con el grupo de pacientes con DTA. Más bien, los pacientes del grupo de demencia mixta sí presentan puntuaciones que se aproximan a las obtenidas por los del grupo de DTA, encontrándose muchas similaridades en los déficit observados en la curva de aprendizaje y en el desempeño neurológico en sus funciones de codificación, ejecutivas y de evocación.

Uno de los aportes de este trabajo es que se puede confirmar la sensibilidad del Test de Aprendizaje Auditivo-Verbal de Rey para el diagnóstico diferenciado entre los casos, no solo de pacientes sanos y con DTA, sino también entre pacientes con demencia vascular y los que presentan DTA. De ese modo, se podría detectar estadios tempranos de la DTA incluso mucho tiempo antes de que pueda establecerse su diagnóstico como tal. La detección temprana permitiría trabajar con los pacientes en intervenciones tanto a nivel neuropsicológico como farmacológico, de manera que permitan ralentizar e, incluso, remitir el desarrollo de la DTA. 
Un trabajo preliminar realizado por tres investigadoras de la Universidad Central de Venezuela menciona que el Test de Aprendizaje Audioverbal de Rey fue el que, a diferencia de otros instrumentos aplicados, discriminó ambos grupos (pacientes con deterioro cognitivo leve y pacientes con DTA), específicamente en el intento VII, e índices V-VII y I-VI (Ferreira, Campagna, y Colmenares, 2008). Así, el estudio arrojó que los sujetos que están propensos a desarrollar DTA presentan alteraciones en el funcionamiento cognitivo, principalmente en las funciones: aprendizaje verbal, memoria de reconocimiento, capacidad de evocación; siendo el Test Auditivo Verbal de Rey la prueba más sensible para detectar tales alteraciones (Ferreira, Campagna, y Colmenares, 2008), lo cual se corresponde con las investigaciones realizadas por Petersen et al. (2001) y, más específicamente, por Otero y Scheitter (2002), quienes afirman que esta prueba es una de las más sensibles para detectar estas tendencias.

El presente estudio es una primera aproximación de las relaciones que se pueden establecer entre la memoria auditiva y el rendimiento neuropsicológico en pacientes de la tercera edad sanos o que presentan alguna de las tres demencias mencionadas en líneas anteriores. Es una apreciación, además, sobre la diferenciación observada en cada uno de los diez ensayos de la memoria auditiva, entre los cuatro grupos de pacientes según el diagnóstico de demencia o condición de sano que tienen. Una limitación que se podría señalar es que el instrumento que se utilizó para medir las evocaciones de los pacientes en cada uno de los ensayos posiblemente no permitiría la detección de variaciones de respuesta ligeras, como sucede en las puntuaciones obtenidas en memoria auditiva por pacientes con demencia tipo Alzheimer y mixta. Los resultados en memoria auditiva entre ambos grupos son bastante similares y sería necesaria una mayor precisión en la determinación de la sensibilidad y especificidad del Test de Aprendizaje Auditivo-Verbal de Rey.

\section{CONCLUSIONES}

Se puede concluir, en términos generales, que existe relación positiva entre la memoria auditiva y el rendimiento neuropsicológico en pacientes de la tercera edad de la muestra investigada. De modo específico, se observa también una relación positiva entre cada una de las dimensiones de la memoria auditiva y el rendimiento neuropsicológico. Finalmente, en cuanto a las comparaciones estadísticas realizadas, se concluye que existen diferencias significativas en las puntuaciones medias obtenidas en cada una de las dimensiones de memoria auditiva, de acuerdo al diagnóstico de demencia, en los pacientes de la muestra estudiada. Además, se encontró que existen diferencias significativas en las puntuaciones medias globales en memoria auditiva, de acuerdo al diagnóstico de demencia, en los pacientes de la tercera edad del estudio. 


\section{REFERENCIAS BIBLIOGRÁFICAS}

Reuter-Lorenz, P. (2002). Neuropsicología cognitiva del cerebro envejecido. En: D. Park y N. Schwarz (Eds.), Envejecimiento Cognitivo. Madrid, España: Médica Panamericana.

Visser, P., Verhey, F., Hofman, P., Scheltens, P., Jolles, J. (2002). Medial temporal lobe atrophy predicts Alzheimer's disease in patients with minor cognitive impairment. Journal of Neurology, Neurosurgery and Psychiatry, 72, 491-497. doi:10.1136/jnnp.72.4.491

Craik, F., \& Jennings, J. (1992). Human memory. En: F. Craik \& Salthouse (Eds.), The Handbook of Aging and Cognition. Hillsdale, NJ: Lawrence Erlbaum Associates.

Kramer, J., Jurik, J., Sha, S. et al. (2003). Distinctive neuropsychological patterns in frontotemporal dementia, semantic dementia, and Alzheimer disease. Cogn Behav Neurol, 16, 211-218. PMID: 14665820

Hernández, R., Fernández, C., Baptista, P. (2010). Metodología de la investigación. México DF, México: McGraw-Hill Interamericana.

Rey, A. (1964). L'examen clinique en Psychologia. Paris, France: Pressee Universitaires de France.

Ostrosky, F., Ardila, A., Rosselli, M. (1999). NEUROPSI: A brief neuropsychological test battery in Spanish. Journal of the International Neuropsychological Society, 5, 413-433.

Gramunt, N. (2008). Normalización y validación de un test de memoria en envejecimiento normal, deterioro cognitivo leve y enfermedad de Alzheimer. Tesis doctoral. Facultat de Psicologia, Ciències de l'Educació i de l'Esport, Blanquerna. Recuperado de http://www.tdx.cat/bitstream/handle/10803/9261/NinaGramunt-tesis.pdf? sequence $=1$

Ferreira, A., Campagna, I., Colmenares, M. (2008). Indicadores neuropsicológicos de evolución a demencia tipo Alzheimer en pacientes diagnosticados con deterioro cognitivo leve. Artículos Segunda Época, 27(2), 9-33. Recuperado de http://saber.ucv.ve/ojs/index.php/rev_ps/article/ view/6277/6041

Petersen, R. et al. (2001). Practice parameter: Early detection of dementia: Mild cognitive impairment (an evidence-based review). Neurology, 56, 1133-1142. PMID: 11342677

Otero, J. y Scheitler, L. (2003). Las fronteras entre el envejecimiento cognitivo normal y la Enfermedad de Alzheimer. El concepto de Deterioro Cognitivo Leve. Revista Médica de Uruguay, 19, 4-13. Recuperado de http:// www.rmu.org.uy/revista/2003v1/art2.pdf 